\title{
Diabetes is associated with increased risk of hepatocellular carcinoma in non-alcoholic steatohepatitis with cirrhosis- implications for surveillance and future pharmacotherapy
}

\author{
Michael P. Johnston ${ }^{1}$, Janisha Patel ${ }^{1}$, Christopher D. Byrne ${ }^{2,3}$ \\ ${ }^{1}$ Department of Hepatology, University Hospital Southampton NHS Foundation Trust, Southampton, UK; ${ }^{2}$ Human Development and Health, \\ Faculty of Medicine, University of Southampton, Southampton, UK; ${ }^{3}$ National Institute for Health Research Southampton Biomedical Research \\ Centre, University of Southampton and University Hospital Southampton NHS Foundation Trust, Southampton, UK \\ Correspondence to: Michael P. Johnston. Department of Hepatology, University Hospital Southampton NHS Foundation Trust, Southampton, UK. \\ Email: michael.johnston1@nhs.net. \\ Provenance and Peer Review: This article was commissioned by the Editorial Office, Hepatobiliary Surgery and Nutrition. The article did not undergo \\ external peer review. \\ Comment on: Yang JD, Ahmed F, Mara KC, et al. Diabetes is associated with increased risk of hepatocellular carcinoma in cirrhosis patients with \\ nonalcoholic fatty liver disease. Hepatology 2019. [Epub ahead of print].
}

Submitted Sep 24, 2019. Accepted for publication Oct 15, 2019.

doi: 10.21037/hbsn.2019.10.09

View this article at: http://dx.doi.org/10.21037/hbsn.2019.10.09

Hepatocellular carcinoma (HCC) is the most common liver-related complication seen in patients with nonalcoholic fatty liver disease (NAFLD) and non-alcoholic steatohepatitis (NASH) (1). NASH is strongly associated with type 2 diabetes mellitus (T2DM) but it has also been apparent for a number of years that T2DM is associated with an increased risk of HCC independent of the presence of NASH (2). A 2006 meta-analysis of retrospective cohort studies and case-control studies demonstrated an association between T2DM and an increased risk of HCC (3). International guidelines advocate surveillance for HCC in patients with NASH who have progressed to cirrhosis (4). An important clinical question is whether T2DM confers an increased risk of HCC once patients with NASH have progressed to cirrhosis. In a recent study by Yang and colleagues (5), the authors reported strong evidence that T2DM is an independent risk factor for HCC in patients with NASH-related cirrhosis. Yang and colleagues performed a retrospective analysis of a prospectively registered research dataset of patients with NASH-related cirrhosis.

It is important to emphasize that the patients in the study by Yang and colleagues all had a diagnosis of cirrhosis and NASH. Cirrhosis was defined by at least one of the following: (I) liver histology consistent with cirrhosis;
(II) clinical features of portal hypertension or hepatic encephalopathy; (III) radiological evidence including a nodular liver; (IV) increased liver stiffness $(>5 \mathrm{kPa}$ on magnetic resonance elastography). The dataset consisted of 354 patients who were seen at the Mayo Clinic Rochester between January 2006 and December 2015. The mean age of the cohort was 61.5 years, $41 \%$ of the patients were male and $71 \%$ of the patients had diabetes, $95 \%$ were white. The median follow-up period was 46 months for patients with T2DM and 47 months for patients without T2DM. The T2DM group was proportionally less white in ethnicity (92.1\% vs. $99.0 \%$ respectively), had lower bilirubin, had lower Child-Pugh score, but had more hypertension and dyslipidaemia. There was a trend towards more cigarette smoking in the T2DM group.

The magnitude of increased risk of HCC in patients with T2DM was modest but significant. The multivariate analysis demonstrated that in patients with NASH-related cirrhosis T2DM was an independent risk factor for the development of HCC with an adjusted hazard ratio (aHR): 4.18, 95\% confidence interval (CI): 1.23-14.2. The multivariate analysis adjusted for all variables for which $\mathrm{P}<0.2$ in the univariate analysis-these were age, sex, ethnicity, ChildPugh score, albumin, platelets and metformin use.

There were 30 cases of HCC (27 in T2DM group and 3 
in group without T2DM). The authors acknowledge that the small number of cases of HCC is a limitation of the study. However, to account for this Yang and colleagues went on to validate the association between T2DM and risk of HCC in 6,630 liver transplant registrants with NASHrelated cirrhosis identified within the United Network for Organ Sharing/Organ Procurement and Transplantation Network (UNOS/OPTN) dataset from between $1^{\text {st }}$ January 2003 and $31^{\text {st }}$ December 2016. The authors demonstrated in the UNOS dataset that T2DM was an independent risk factor for HCC in patients with NASH-cirrhosis (aHR: 1.38, 95\% CI: 1.19-1.59). The multivariate analysis adjusted for all variables for which $\mathrm{P}<0.2$ in the univariate analysis - these were age, sex, Child-Pugh score, albumin and creatinine. There are limitations in analysis of the UNOS registry, particularly with regard to removal of patients from the registry. A previous study of data from four transplant centres demonstrated that the UNOS registry misclassified $8.6 \%$ of patients removed for death or clinical deterioration-they were assigned the code 'other' (6). Nearly half of the $8.6 \%$ of patients misclassified as 'other' were in fact removed due to development of HCC. The authors acknowledged a further limitation of the UNOS dataset which was the potential removal of patients who develop HCC outwith Milan criteria but who were censored from the analysis due to their removal. These censored patients would not have been counted as cases of HCC. These stated limitations of the UNOS registry may also account for the lower aHR observed compared to the Mayo Clinic Rochester cohort.

In the study by Yang and colleagues obesity was not associated with an increased risk of HCC. Obesity has been previously identified as a risk factor for the development of HCC in the general population (7). It is interesting that Yang and colleagues did not find an association between obesity and risk of development of HCC. However, it is possible that the apparent lack of association relates to the methodology in the study by Yang and colleagues. Firstly, by virtue of being a cirrhotic population there was presumably a number of patients with ascites or hypoalbuminaemia associated oedema. The presence of ascites or oedema confounds the conventional definition of obesity based on body mass index (BMI). Secondly, an increased risk of HCC is most strongly associated with early adult obesity (8). A history of early adult obesity was not used in the study by Yang and colleagues.

Contrary to previous suggestions elsewhere that metformin may reduce the risk of development of HCC (9),
Yang and colleagues found that the use of metformin was not associated with an altered risk of HCC in patients with NASH-related cirrhosis. However, the authors acknowledge the limitation which was the small number of patients on metformin in the study. Treatment with metformin has previously been associated with a $~ 50 \%$ decrease in incidence of HCC in patients with NAFLD when compared to incidence of HCC in patients treated with sulphonylureas, glitazones or insulin (10). Furthermore, a protective effect of metformin against HCC development seems to have biological plausibility. As illustrated in Figure 1, metformin is associated with alteration of the gut microbiota, and in a mouse model there is a particular increase in number of the bacterium Akkermansia (14). Oral administration of Akkermansia to high fat diet mice without metformin improves glucose tolerance and reduces adipose tissue inflammation (14). The possibility of an effect of insulin on HCC risk in T2DM is less clear. There has been a previous in vitro study of human hepatic stellate cells which demonstrated that insulin stimulated hepatic stellate cell proliferation and collagen synthesis in vitro (15). This might suggest a potential mechanism by which exogenous insulin could promote liver fibrosis and hepatocyte proliferation leading to HCC. However, the difficulty in analysing previous observational studies for the association between metformin and HCC risk, or insulin and HCC risk, is that therapy for T2DM changes with severity of T2DM. Therefore, severity of T2DM is likely to be a confounder.

The finding that $\mathrm{T} 2 \mathrm{DM}$ is an independent risk factor for HCC even in the presence of NASH and liver cirrhosis supports a proposal that there may be additional mechanisms promoting hepatocarcinogenesis. Some of the mechanisms by which T2DM appears to promote hepatocarcinogenesis are mediated via changes in the gut wall (see Figure 1). The intestinal dysbiosis which is associated with T2DM appears to have an effect on the integrity of the gut wall (16). This increased permeability of the gut wall allows direct translocation into the portal vein of pro-inflammatory bacterial components such as lipopolysaccharide (LPS), which has been shown to promote tumorigenesis in a mouse model via the toll-like receptor 4 (TLR-4) (17). There is also the potential effect of change in concentrations of short chain fatty acid (SCFA) in T2DM. In T2DM there is a reduction in production of SCFAs such as butyrate and propionate due to fiberpoor diet and altered profile of the microbiota (16). In an animal model propionate appears to normally downregulate 


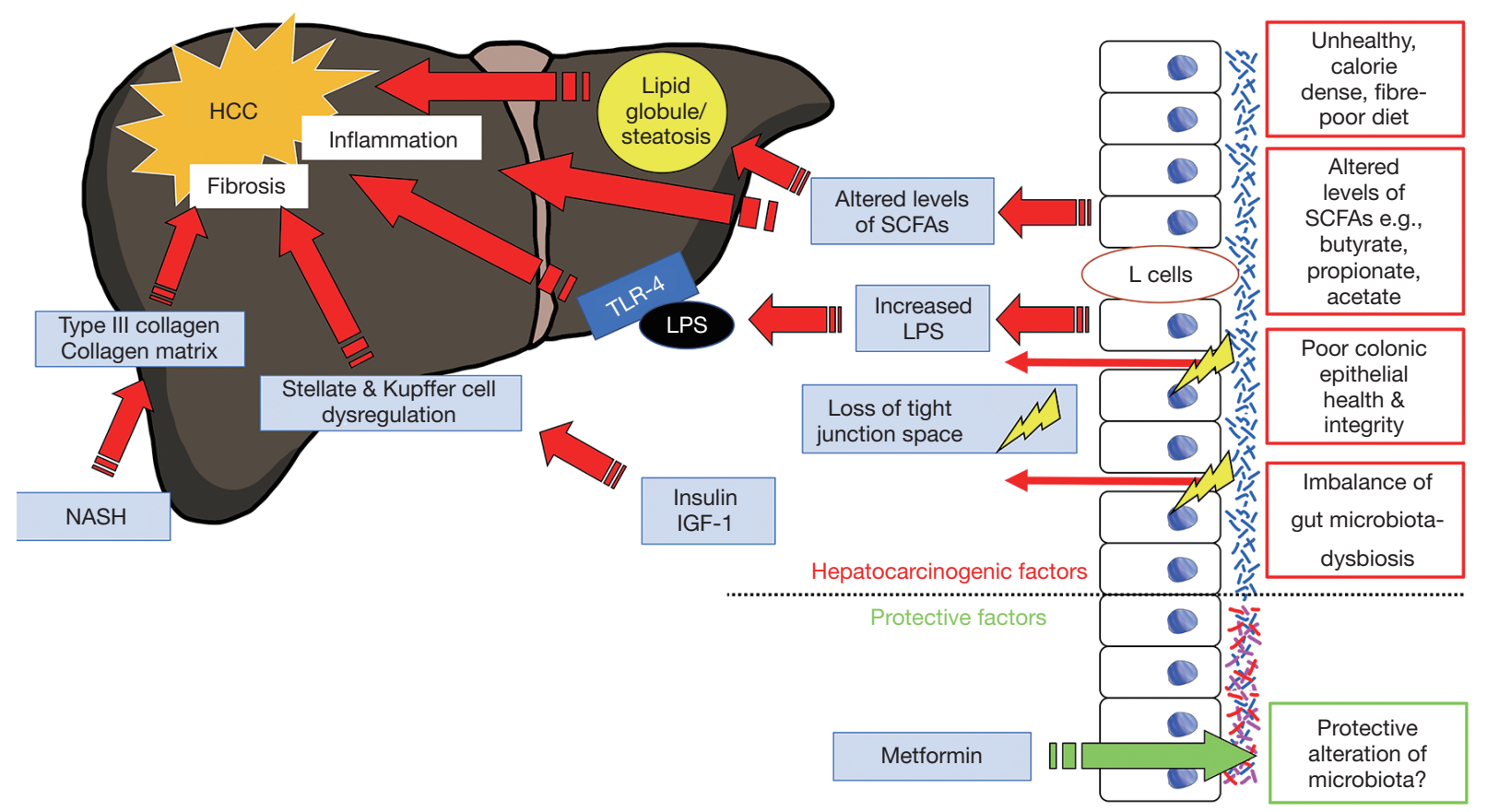

Figure 1 Mechanistic pathways associated with promotion of hepatocarcinogenesis in T2DM. T2DM is associated with an increased risk of NASH. NASH is associated with a progressive process of collagen deposition and fibrogenesis. This inflammatory process drives hepatocellular DNA damage, endoplasmic reticulum stress and hepatocellular necrosis leading to regenerative nodule formation, dysplastic nodules and ultimately carcinoma. Unhealthy diet and T2DM-related dysbiosis within the gut are associated with loss of intestinal integrity and altered levels of SCFAs. Due to loss of intestinal integrity there is translocation of pro-inflammatory bacterial components such as LPS across the gut wall, which activates TLR-4 receptors. There is evidence that TLR-4-dependent secretion of growth factors, including epiregulin, mediate a hepatocarcinogenic effect (11). Butyrate is a SCFA that upregulates apoptosis in the liver via modulation of ROS. The alteration in levels of SCFAs, including butyrate, is associated with reduced apoptosis and increased steatosis, cholestasis and hepatocarcinogenesis (12). In the presence of insulin resistance and NASH there is also a reduction in levels of IGF-1 (13). Alteration in IGF-1 levels may dysregulate immune response by HSCs, predisposing to hepatocellular proliferation and hepatocarcinogenesis. Insulin appears to stimulate HSCs collagen deposition. T2DM, type 2 diabetes mellitus; NASH, non-alcoholic steatohepatitis; SCFA, short chain fatty acid; LPS, lipopolysaccharide; TLR-4, toll-like receptor 4; ROS, reactive oxygen species; IGF-1, insulin-like growth factor 1; HSC, hepatic stellate cell; HCC, hepatocellular carcinoma.

malignant cell proliferation in the liver (18).

Yang and colleagues have demonstrated that T2DM is an independent risk factor for the development of HCC in the most severe form of NAFLD, i.e., NASH-related cirrhosis. The authors have made reference to the potential clinical implications of this finding. Firstly, it may better help identify those at risk of HCC such that more sensitive surveillance strategies may be developed. Secondly, it may identify modifiable risk factors which could be the target of interventions or pharmacotherapy, and therefore reduce overall risk of developing HCC.

With regards to the first issue of surveillance or monitoring for HCC in NASH, the authors conclude that 'bigh-risk patients such as older diabetics with low serum albumin level should be carefully monitored for HCC development'. However, all of the patients in the study by Yang and colleagues had cirrhosis. International guidelines (4) currently recommend surveillance for HCC in patients with cirrhosis (regardless of the presence of other risk factors). There is not an accepted strategy for monitoring for HCC in non-cirrhotic patients (19). This is a source of debate because HCC develops in non-cirrhotic patients with NASH (20) and the risk of HCC is greater in non-cirrhotic $\mathrm{NASH}$ patients relative to non-cirrhotic patients of other 
aetiologies (21).

With regards to modifiable risk factors, Yang and colleagues also mention the lack of association between risk of development of HCC and metformin use in their study. However, the authors were unable to provide data on HbA1c or duration of T2DM. Therefore, in the absence of a well-matched control incorporating these characteristics it is difficult to draw a conclusion about whether metformin attenuates HCC risk or not.

It is important to consider the effect of potential pharmacotherapy in T2DM and the effect it may have on risk of development of HCC in NASH-related cirrhosis. Downregulation of the mechanisms contributing to HCC risk in NASH will also be challenging for any future single agent licensed for NASH. The current drugs in development for NASH primarily aim to improve fibrosis stage, not the metabolic profile (22). Yang and colleagues have demonstrated that T2DM is also an independent risk factor for development of HCC in NASH-related cirrhosis. We agree with the authors that prospective studies are needed to examine the effect of agents for T2DM and their effect on HCC risk.

\section{Acknowledgments}

Funding: Christopher D. Byrne is supported in part by grants from the Southampton National Institute for Health Research Biomedical Research Centre, Southampton, UK.

\section{Footnote}

Conflicts of Interest: All authors have completed the ICMJE uniform disclosure form (available at https://hbsn. amegroups.com/article/view/10.21037/hbsn.2019.10.09/coif). The authors have no conflicts of interest to declare.

Ethical Statement: The authors are accountable for all aspects of the work in ensuring that questions related to the accuracy or integrity of any part of the work are appropriately investigated and resolved.

Open Access Statement: This is an Open Access article distributed in accordance with the Creative Commons Attribution-NonCommercial-NoDerivs 4.0 International License (CC BY-NC-ND 4.0), which permits the noncommercial replication and distribution of the article with the strict proviso that no changes or edits are made and the original work is properly cited (including links to both the formal publication through the relevant DOI and the license). See: https://creativecommons.org/licenses/by-nc$\mathrm{nd} / 4.0 /$.

\section{References}

1. Akuta N, Kawamura Y, Arase Y, et al. Hepatocellular carcinoma is the most common liver-related complication in patients with histopathologically-confirmed NAFLD in Japan. BMC Gastroenterol 2018;18:165.

2. Koh WP, Wang R, Jin A, et al. Diabetes mellitus and risk of hepatocellular carcinoma: findings from the Singapore Chinese Health Study. Br J Cancer 2013;108:1182-8.

3. El-Serag HB, Hampel H, Javadi F. The association between diabetes and hepatocellular carcinoma: a systematic review of epidemiologic evidence. Clin Gastroenterol Hepatol 2006;4:369-80.

4. Marrero JA, Kulik LM, Sirlin CB, et al. Diagnosis, staging, and management of hepatocellular carcinoma: 2018 practice guidance by the American Association for the Study of Liver Diseases. Hepatology 2018;68:723-50.

5. Yang JD, Ahmed F, Mara KC, et al. Diabetes is associated with increased risk of hepatocellular carcinoma in cirrhosis patients with nonalcoholic fatty liver disease. Hepatology 2019. [Epub ahead of print].

6. Goldberg D, French B, Trotter J, et al. Underreporting of liver transplant waitlist removals due to death or clinical deterioration: results at four major centers. Transplantation 2013;96:211-6.

7. Schlesinger S, Aleksandrova K, Pischon T, et al. Abdominal obesity, weight gain during adulthood and risk of liver and biliary tract cancer in a European cohort. Int J Cancer 2013;132:645-57.

8. Hassan MM, Abdel-Wahab R, Kaseb A, et al. Obesity early in adulthood increases risk but does not affect outcomes of hepatocellular carcinoma. Gastroenterology 2015;149:119-29.

9. Schulte L, Scheiner B, Voigtländer T, et al. Treatment with metformin is associated with a prolonged survival in patients with hepatocellular carcinoma. Liver Int 2019;39:714-26.

10. Singh S, Singh PP, Singh AG, et al. Anti-diabetic medications and the risk of hepatocellular cancer: a systematic review and meta-analysis. Am J Gastroenterol 2013;108:881-91; quiz 892.

11. Dapito DH, Mencin A, Gwak GY, et al. Promotion of hepatocellular carcinoma by the intestinal microbiota and TLR4. Cancer Cell 2012;21:504-16. 
12. Singh V, Yeoh BS, Chassaing B, et al. Dysregulated microbial fermentation of soluble fiber induces cholestatic liver cancer. Cell 2018;175:679-94.e22.

13. Yao Y, Miao X, Zhu D, et al. Insulin-like growth factor-1 and non-alcoholic fatty liver disease: a systemic review and meta-analysis. Endocrine 2019;65:227-37.

14. Shin NR, Lee JC, Lee HY, et al. An increase in the Akkermansia spp. population induced by metformin treatment improves glucose homeostasis in diet-induced obese mice. Gut 2014;63:727-35.

15. Svegliati-Baroni G, Ridolfi F, Di Sario A, et al. Insulin and insulin-like growth factor-1 stimulate proliferation and type I collagen accumulation by human hepatic stellate cells: differential effects on signal transduction pathways. Hepatology 1999;29:1743-51.

16. Tilg H, Moschen AR. Microbiota and diabetes: an evolving relationship. Gut 2014;63:1513-21.

17. Ponziani FR, Bhoori S, Castelli C, et al. Hepatocellular carcinoma is associated with gut microbiota profile and inflammation in nonalcoholic fatty liver disease.

Cite this article as: Johnston MP, Patel J, Byrne CD. Diabetes is associated with increased risk of hepatocellular carcinoma in non-alcoholic steatohepatitis with cirrhosis-implications for surveillance and future pharmacotherapy. HepatoBiliary Surg Nutr 2020;9(2):230-234. doi: 10.21037/hbsn.2019.10.09
Hepatology 2019;69:107-20.

18. Bindels LB, Porporato P, Dewulf EM, et al. Gut microbiota-derived propionate reduces cancer cell proliferation in the liver. Br J Cancer 2012;107:1337-44.

19. Younes R, Bugianesi E. Should we undertake surveillance for HCC in patients with NAFLD? J Hepatol 2018;68:326-34.

20. Paradis V, Zalinski S, Chelbi E, et al. Hepatocellular carcinomas in patients with metabolic syndrome often develop without significant liver fibrosis: a pathological analysis. Hepatology 2009;49:851-9.

21. Stine JG, Wentworth BJ, Zimmet A, et al. Systematic review with meta-analysis: risk of hepatocellular carcinoma in non-alcoholic steatohepatitis without cirrhosis compared to other liver diseases. Aliment Pharmacol Ther 2018;48:696-703.

22. Johnston MP, Patel J, Byrne CD. Multi-drug approaches to NASH: what's in the development pipeline? Expert Opin Investig Drugs 2020;29:143-50. 